\title{
THERMODYNAMICS ANALYSIS FOR A REACTING COUPLE STRESS FLUID FLOW THROUGH A VERTICAL CHANNEL
}

\author{
Samuel O. Adesanya ${ }^{1,2} \S$, J.A. Falade ${ }^{3}$, \\ J.C. Ukaegbu ${ }^{1}$, R.S. Lebelo ${ }^{2}$ \\ ${ }^{1}$ Department of Mathematical Sciences \\ Redeemer's University \\ Redemption City, NIGERIA \\ ${ }^{2}$ Department of Mathematics \\ Vaal University of Technology \\ Private Bag X021, Vanderbijlpark, 1911, SOUTH AFRICA \\ ${ }^{3}$ Department of Physical Sciences \\ Redeemer's University \\ Redemption City, NIGERIA
}

\begin{abstract}
In this paper, entropy generation rate in the flow of couple stress fluid undergoing exothermic chemical reaction through a porous vertical channel is studied by applying the second law of thermodynamics. A semi-analytical solution for the governing equations is obtained using Adomian decomposition method (ADM). The effects of couple stress parameter, internal heat generation parameter, Dufour number on the velocity, temperature distribution, entropy generation rate and Bejan number are investigated and the results were presented graphically. The results show that solutal diffusivity has significant effect on the entropy generation rate and irreversibility of heat in a couple stress fluid flow.
\end{abstract}

Key Words: entropy generation, couple stresses, double diffusivity, ADM, irreversibility ratio

Received: $\quad$ September 26, 2016

Revised: May 11, 2017

Published: $\quad$ May 23, 2017

$\S_{\text {Correspondence author }}$ (c) 2017 Academic Publications, Ltd. url: www.acadpubl.eu 


\section{Introduction}

One striking property of the fluid undergoing exothermic chemical reaction is the heat released due to of fluid particles interaction. The heat released could be mild or explosive depending on the concentration of the reacting species. Hence, thermodynamics plays a major role in the dynamics of reacting fluid. Based on the heat generation, chemical reactions are induced in many chemical engineering processes to get a desired product. Classical examples in the petrochemical industry and chemical engineering includes; the in-situ combustion of heavy hydrocarbon during exploration, production of hydrogen energy by oxidation of butanols. Other applications of chemical interactions in heat and mass transfer could be seen in the polymerization of fluids like polyethylene oxide and poly isobutylene solutions.

Over the years, the thermodynamics of flow and heat transfer has been based on the second law analysis and its design-related concept of entropy generation minimization. The entropy generation and efficiency calculation using the second law of thermodynamics are more reliable than first law-based calculations. The analysis of entropy generation minimization in a thermal system was introduced by Bejan [1] and explained that this minimization improves the efficiency of a system by improving the exergy. Thereafter, several researchers have theoretically studied entropy generation in thermal and flow systems under many physical situations [2-8].

The majority of the studies reported in the literature dealt with the traditional Newtonian fluids. Several fluids used in engineering and industrial processes, such as suspensions of particles and fibers, slurries, polymer solutions and melts, surfactants, paints, foodstuffs, body fluids, soaps, inks, organic materials, adhesives, etc., exhibit flow properties that cannot be explained by Newtonian fluid flow model. A number of mathematical models have been proposed to explain the rheological behaviour of these fluids. One among these models is couple stress fluid model introduced by Stokes. The couple stress fluid model has distinct features, such as the presence of couple stresses, body couples and non-symmetric stress tensor. The couple stress fluid theory presents models for fluids whose microstructure is of mechanical significance. These fluids exhibit more complicated size dependant effect than those in Newtonian fluids. Several researchers have studied analytically and mostly numerically the convective flow of couple stress fluid through a vertical channel. Makinde and Eegunjobi [9] studied the inherent irreversibility in a steady flow of a couple stress fluid through a vertical channel packed with saturated porous substances. Makinde and Adesanya [10] investigated the entropy generation in couple stress 
fluid flowing steadily through a porous channel filled with slip at the isothermal walls. Adesanya and Makinde [11] examined the effect of couple stresses on the entropy generation rate of an incompressible viscous fluid through a porous channel with convective heating at the walls. Recently, Srinivasacharya and Kaladhar [12] considered the Soret and Dufour effects in the presence of chemical reaction on natural convection heat and mass transfer of a couple stress fluid in a vertical channel.

Motivated by the investigations and applications mentioned above, the aim of this investigation is to consider the entropy generation and irreversibility of heat based on the second law of thermodynamics so as to measure the exergy of chemically reacting couple stress fluids. It may be remarked that earlier studies, to the best of the authors knowledge, did not include the effect of chemical reaction and Dufour term on entropy generation rate in the flow couple stress fluid through a vertical channel. The problem addressed here is a fundamental one that arises in the chemical industry where reacting couple stress fluid is the working medium. The resulting coupled nonlinear differential equations are solved using a semi-analytical method, i.e., Adomian decomposition method. The choice of this non-perturbative method is due to the fact that, it requires no discretization, initial guess, linearization or any simplifying assumptions. The method has been applied to several linear and non-linear problems in recent times, see ([13]-[17]). The approximate solutions are used to compute the entropy generation rate and the irreversibility analysis.

\section{Mathematical Analysis}

Consider the steady free convective flow of a couple stress fluid between two permeable vertical plates of distance $2 d$ apart. The $x$-axis be taken along vertically upward direction through the central line of the channel and $y$-axis is perpendicular to the plates. The plate $y=-d$ has a uniform temperature $T_{0}$ together with concentration $C_{0}$. On the other hand, at the plate $y=d$ the fluid has a uniform temperature $T_{1}$ and concentration $C_{1}$. It is assumed that the physical quantities depend on $y$ only since the flow is fully developed. All the fluid properties are assumed to be constant except for density and internal heat generation which depends linearly on temperature. Under the usual Bousinesq approximation, the equations governing the fluid flow and entropy generation rate can be written as

$$
\rho \nu_{0} \frac{d u^{\prime}}{d y^{\prime}}=\mu \frac{d^{2} u^{\prime}}{d y^{2^{\prime}}}-\eta \frac{d^{4} u^{\prime}}{d y^{4^{\prime}}}+\rho g \beta_{1}\left(T-T_{0}\right)+\rho g \beta_{C}\left(C-C_{0}\right),
$$




$$
\begin{aligned}
\rho C_{p} \nu_{0} \frac{d T}{d y^{\prime}}= & k \frac{d^{2} T}{d y^{\prime 2}}+\mu\left(\frac{d u^{\prime}}{d y^{\prime}}\right)^{2}+\eta\left(\frac{d^{2} u^{\prime}}{d y^{2^{\prime}}}\right)+D_{m}\left(\frac{d^{2} C}{d y^{2^{\prime}}}\right)+Q_{0}\left(T-T_{0}\right) \\
\nu_{0} \frac{d C}{d y^{\prime}}= & D_{m} \frac{d^{2} C}{d y^{\prime 2}}-Q_{1}\left(C-C_{0}\right) \\
E_{0}= & \frac{k}{T_{0}^{2}}\left(\frac{d T}{d y^{\prime}}\right)^{2}+\frac{\mu}{T_{0}}\left(\frac{d u^{\prime}}{d y^{\prime}}\right)^{2}+\frac{\eta}{T_{0}}\left(\frac{d^{2} u^{\prime}}{d y^{\prime 2}}\right)^{2} \\
& +\frac{D_{M}}{T_{0}\left(C_{1}-C_{0}\right)}\left(\frac{d^{2} C}{d y^{\prime 2}}\right)^{2}
\end{aligned}
$$

The appropriate boundary conditions for the uniform wall temperature are

$$
\begin{aligned}
& u^{\prime}=\frac{d^{2} u^{\prime}}{d y^{\prime 2}}=0, T=T_{0}, C=C_{0} \text { on } y=-h, \\
& u^{\prime}=\frac{d^{2} u^{\prime}}{d y^{\prime 2}}=0, T=T_{1}, C=C_{1} \text { on } y=h .
\end{aligned}
$$

Introducing the following dimensionless variables and parameters

$$
\begin{gathered}
y=\frac{y^{\prime}}{h}, u=\frac{u^{\prime}}{\nu_{0}}, \theta=\frac{T^{\prime}-T_{0}}{T_{f}-T_{0}}, \\
R=\frac{\nu_{0} h}{\nu}, \lambda^{2}=\frac{\mu h^{2}}{\eta}, S c=\frac{\nu}{D_{m}}, \gamma=\frac{Q_{1} h^{2}}{D_{m}}, \\
\operatorname{Pr}=\frac{\mu C_{p}}{k}, B r=\frac{\nu_{0}^{2} \mu}{k\left(T_{1}-T_{0}\right)}, G r_{T}=\frac{g \beta_{T} h^{2}\left(T_{1}-T_{0}\right)}{\nu_{0} \nu}, \delta=\frac{Q_{0} h^{2}}{k}, \\
G r_{C}=\frac{g \beta_{T} h^{2}\left(C_{1}-C_{0}\right)}{\nu_{0} \nu}, \\
\Gamma=\frac{T_{0}^{2} h^{2} E_{0}}{k\left(T_{f}-T_{0}\right)^{2}}, \Omega=\frac{T_{1}-T_{0}}{T_{0}}, D_{f}=\frac{D_{m}\left(C_{1}-C_{0}\right)}{k\left(T_{1}-T_{0}\right)} .
\end{gathered}
$$

We have, the following dimensionless equations

$$
\begin{aligned}
R \frac{d u}{d y} & =G+\frac{d^{2} u}{d y^{2}}-\frac{1}{\lambda^{2}} \frac{d^{2} u}{d y^{4}}+G r_{1} \theta+G r_{C} \varphi \\
R P r \frac{d \theta}{d y} & =\frac{d^{2} \theta}{d y^{2}}+B r\left(\frac{d u}{d y}\right)^{2}+\frac{B r}{\lambda^{2}}\left(\frac{d^{2} u}{d y^{2}}\right)^{2}+D_{f} \frac{d^{2} \varphi}{d y^{2}}+\delta \theta \\
R S C \frac{d \varphi}{d y} & =\frac{d^{2} \varphi}{d y^{2}}-\gamma \varphi
\end{aligned}
$$




$$
\Gamma=\left(\frac{d \theta}{d y}\right)^{2}+\frac{B r}{\Omega}\left(\frac{d u}{d y}\right)^{2}+\frac{B r}{\Omega a^{2}}\left(\frac{d^{2} u}{d y^{2}}\right)^{2}+\frac{D_{f}}{\Omega}\left(\frac{d \varphi}{d y}\right)^{2}
$$

together with appropriate boundary conditions

$$
\begin{aligned}
& u=\frac{d^{2} u}{d y^{2}}=0, \theta=0, \varphi=0 \text { on } y=-1, \\
& u=\frac{d^{2} u}{d y^{2}}=0, \theta=1, \varphi=1 \text { on } y=1 .
\end{aligned}
$$

\section{Method of Solution}

The exact solution of (10) subject to the boundary condition can be written as

$$
\varphi(y)=m_{1}\left(e^{\frac{m_{2}}{2} y}-e^{\frac{m_{1}}{2} y}\right)
$$

where

$$
\begin{gathered}
m_{1}=-\frac{e^{-\frac{R S c}{2}}+\frac{1}{2} \sqrt{R^{2} S c^{2}+4 \gamma}}{-1+e^{\sqrt{R^{2} S c^{2}+4 \gamma}}}, \\
m_{2}=R S c-\sqrt{R^{2} S c^{2}+4 \gamma} \\
m_{3}=R S c+\sqrt{R^{2} S c^{2}+4 \gamma}
\end{gathered}
$$

Using the boundary conditions, we get the coupled integral equations

$$
\begin{aligned}
u(y)= & \int_{-1}^{y} a_{0} d Y+\int_{-1}^{y} \int_{-1}^{y} \int_{-1}^{y} a_{1} d Y d Y d Y \\
& Y+\int_{-1}^{y} \int_{-1}^{y} \int_{-1}^{y} \int_{-1}^{y} \lambda^{2}\left(\frac{d^{2} u}{d Y^{2}}-R \frac{d u}{d Y}+G r_{T} \theta\right. \\
& \left.+G r_{C} \varphi\right) d Y d Y d Y d Y \\
\theta(y)= & \int_{-1}^{y} b_{0} d Y+\int_{-1}^{y} \int_{-1}^{y}\left\{\operatorname{RPr} \frac{d \theta}{d Y}-B r\left(\frac{d u}{d Y}\right)^{2}\right. \\
& \left.-\frac{B r}{\lambda^{2}}\left(\frac{d^{2} u}{d Y^{2}}\right)^{2}+D_{f} \frac{d^{2} \varphi}{d Y^{2}}-\delta \theta\right\} d Y d Y .
\end{aligned}
$$

Now, let us assume a series solution in the form

$$
u(y)=\sum_{n=0}^{\infty} u_{n}(y), \quad \theta(y)=\sum_{n=0}^{\infty} \theta_{n}(y)
$$




$$
\begin{array}{r}
\sum * x_{n=0} u_{n}(y)=\int_{-1}^{y} a_{0} d Y+\int_{-1}^{y} \int_{-1}^{y} \int_{-1}^{y} \int_{-1}^{y} a_{1} d Y d Y d Y \\
+\int_{-1}^{y} \int_{-1}^{y} \int_{-1}^{y} \int_{-1}^{y} \int_{-1}^{y} \lambda^{2}\left(\frac{d^{2}}{d Y^{2}}\left(\sum_{n=0}^{x} u_{n}(y)\right)\right) \\
+\int_{-1}^{y} \int_{-1}^{y} \int_{-1}^{y} \int_{-1}^{y} \int_{-1}^{y} \lambda^{2}\left(G r_{T}\left(\sum_{n=0}^{\infty} \theta_{n}(y)\right)-R \frac{d}{d Y}\left(\sum_{n=0}^{\infty} u_{n}(y)\right)\right. \\
\left.+G r_{C} \varphi(y)\right) d Y d Y d Y d Y,
\end{array}
$$

with

$$
\begin{aligned}
& \sum_{n=0}^{\infty} \theta_{n}(y)=\int_{-1}^{y} b_{0} d Y \\
& \quad+\int_{-1}^{y} \int_{-1}^{y}\left\{\operatorname{RPr} \frac{d}{d Y}\left(\sum_{n=0}^{\infty} \theta_{n}(y)\right)-B r\left(\frac{d}{d Y}\left(\sum_{n=0}^{\infty} u_{n}(y)\right)\right)^{2}\right\} d Y d Y \\
& +\int_{-1}^{y} \int_{-1}^{y}\left\{D_{f} \frac{d^{2} \varphi}{d Y^{2}}-\frac{B r}{\lambda^{2}}\left(\frac{d^{2}}{d Y^{2}}\left(\sum_{n=0}^{\infty} u_{n}(y)\right)\right)^{2}-\delta\left(\sum_{n=0}^{\infty} \theta_{n}(y)\right)\right\} d Y d Y .
\end{aligned}
$$

Then the recursive relation for the coupled problem can be written as

$$
\begin{aligned}
u_{0}(y)= & \int_{-1}^{y} a_{0} d Y+\int_{-1}^{y} \int_{-1}^{y} \int_{-1}^{y} a_{1} d Y d Y d Y \\
& +\int_{-1}^{y} \int_{-1}^{y} \int_{-1}^{y} \int_{-1}^{y}\left(\lambda^{2} G r_{C} \varphi(y)\right) d Y d Y d Y d Y, \\
u_{n+1}(y)= & \int_{-1}^{y} \int_{-1}^{y} \int_{-1}^{y} \int_{-1}^{y} \lambda^{2}\left(\frac{d^{2} u_{n}}{d Y^{2}}-R \frac{d u_{n}}{d Y}\right. \\
& \left.+G r_{T} \theta_{n}+G r_{C} \varphi_{n}\right), d Y d Y d Y d Y
\end{aligned}
$$

together with

$$
\begin{aligned}
\theta(y) & =\int_{-1}^{y} b_{0} d Y-\int_{-1}^{y} \int_{-1}^{y} D_{f} \frac{d^{2} \varphi}{d Y^{2}} d Y d Y, \\
\theta_{n-1}(y) & =\int_{-1}^{y} \int_{-1}^{y}\left\{\operatorname{RPr} \frac{d \theta}{d Y}-\operatorname{Br} A_{n}-\delta \theta_{n}\right\} d Y d Y .
\end{aligned}
$$


The constants $a_{0}, a_{1}, b_{0}$ are obtained using the other boundary conditions. Where the nonlinear term in (21) is given by

$$
A_{n}=\left(\frac{d u_{n}}{d Y}\right)^{2}+\frac{1}{\lambda^{2}}\left(\frac{d^{2} u_{n}}{d Y^{2}}\right)^{2}
$$

and can be expanded as follows

$$
\begin{aligned}
A_{0} & =\left(\frac{d u_{0}}{d Y}\right)^{2}+\frac{1}{\lambda^{2}}\left(\frac{d^{2} u_{0}}{d Y^{2}}\right)^{2}, \\
A_{1} & =2\left(\frac{d u_{0}}{d Y}\right)\left(\frac{d u_{1}}{d Y}\right)+\frac{2}{\lambda^{2}}\left(\frac{d^{2} u_{0}}{d Y^{2}}\right)^{2}\left(\frac{d^{2} u_{1}}{d Y^{2}}\right), \\
A_{2} & =2\left(\frac{d u_{0}}{d Y}\right)\left(\frac{d u_{2}}{d Y}\right)+\left(\frac{d u_{1}}{d Y}\right)^{2} \\
& +\frac{2}{\lambda^{2}}\left(\frac{d^{2} u_{0}}{d Y^{2}}\right)\left(\frac{d^{2} u_{2}}{d Y^{2}}\right)+\frac{1}{\lambda^{2}}\left(\frac{d^{2} u_{1}}{d Y^{2}}\right)^{2} .
\end{aligned}
$$

So that the approximate solution can be written as the truncated series

$$
u(y)=\sum_{n=0}^{m} u_{n}(y), \quad \theta(y)=\sum_{n=0}^{m} \theta_{n}(y), \quad \varphi(y)=\sum_{n=0}^{m} \varphi_{n}(y),
$$

where $m$ represents the number of iterations. Rigorous proof of the convergence of the series solution has been dealt with in [13]-[16] and these theorems will not be repeated here.

To compute the irreversibility ratio for the heat flow, we set

$$
N_{1}=\left(\frac{d \theta}{d y}\right)^{2}, N_{2}=\frac{B r}{\Omega}\left(\frac{d u}{d y}\right)^{2}+\frac{B r}{\Omega a^{2}}\left(\frac{d^{2} u}{d y^{2}}\right)^{2}, N_{3}=\frac{D_{f}}{\Omega}\left(\frac{d \varphi}{d y}\right)^{2} .
$$

Such that the Bejan number $(\Lambda)$ can be written as

$$
\Lambda=\frac{N_{1}}{N_{1}+N_{2}+N_{3}}=\frac{1}{1+\frac{N_{2}+N_{3}}{N_{1}}}=\frac{1}{1+\phi},
$$

where $\phi=\frac{N_{2}+N_{3}}{N_{1}}$.

Observe that the $\Lambda$ is bounded between 0 and 1. This fraction will allow us to investigate the irreversibility due to heat transfer, fluid viscosity and mass transfer. 


\section{Results and Discussion}

Equations (11), (20)-(25) are coded in MATHEMATICA and expression for the constants $a_{0}, a_{1}, b_{0}$ are obtained. Results are presented for various values of the couple stress inverse, internal heat generation parameter and Dufour number. Unless otherwise stated, all parameter values are fixed at unity except for $B r=0.1$. This values have been used to compute the following graphical results.

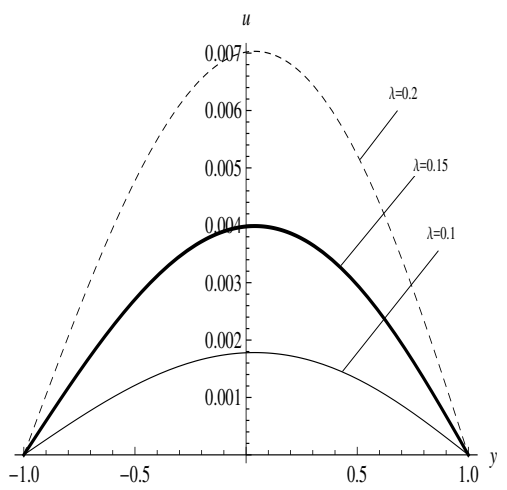

Figure 1: Effect of increasing couple stress inverse parameter on velocity profile

Figures 1-3 shows the effect of different fluid parameter on the flow. From the results, the increasing values of couple stress inverse is observed to increase the fluid flow in Figure 3. This shows that fluid velocity decreases with increase in the non-Newtonian material parameter. Moreover, in Figure 2, it is observed that increase in the internal heat generation due to exothermic nature of the chemical interaction enhances the fluid flow. This is in agreement with many engineering applications especially recovery of heavy fluid. Similar phenomenon is observed with increase in the concentration of the reacting species as observed in Figure 3. Also, in figures 4-5, it is observed that an increase in the Dufour number, couple stress inverse and internal heat generation has increasing effect on the fluid temperature distribution within the channel. This is due to increasing heat liberation resulting from the chemical kinetics of the fluid particles. The entropy generation rate within the channel is presented in figures 7-9. In Figure 6, one observed that entropy generation increases with increase in the couple stress inverse but it is higher at the heated wall than the cold wall. Sim- 


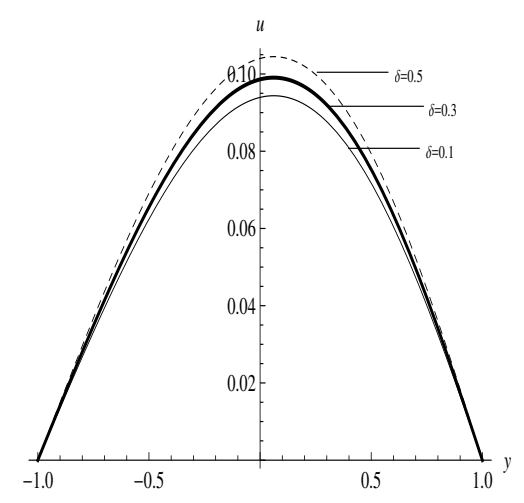

Figure 2: Effect of increasing internal heat generation parameter on velocity profile

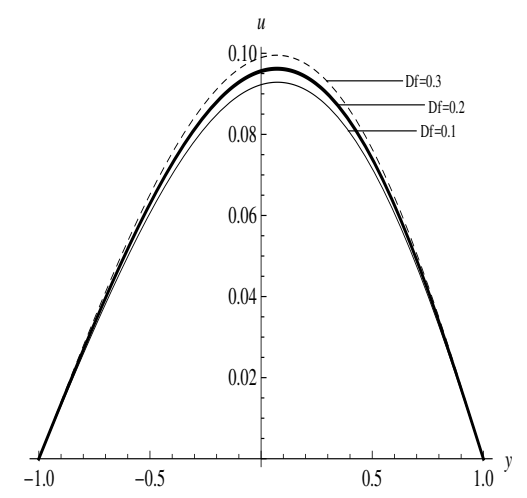

Figure 3: Effect of increasing Dufour number on velocity profile 


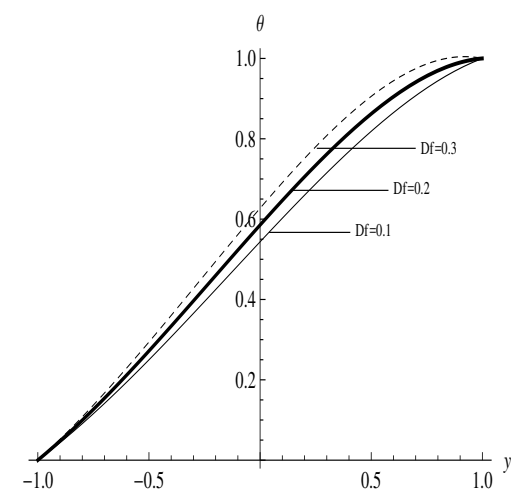

Figure 4: Effect of increasing Dufour number on temperature profile

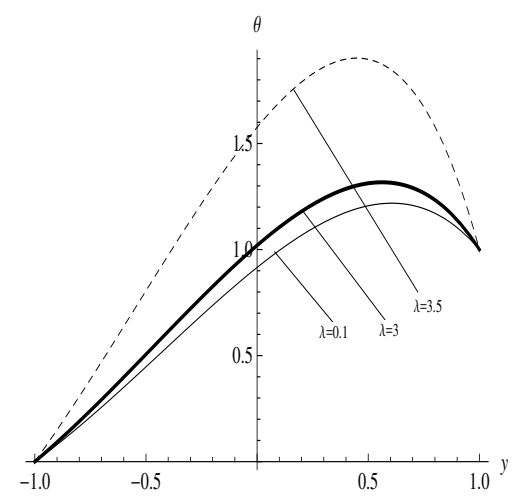

Figure 5: Effect of increasing couple stress inverse parameter on temperature profile 


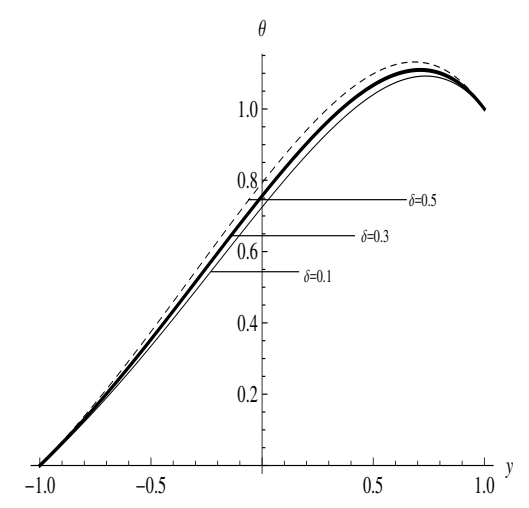

Figure 6: Effect of increasing internal heat generation parameter on temperature profile

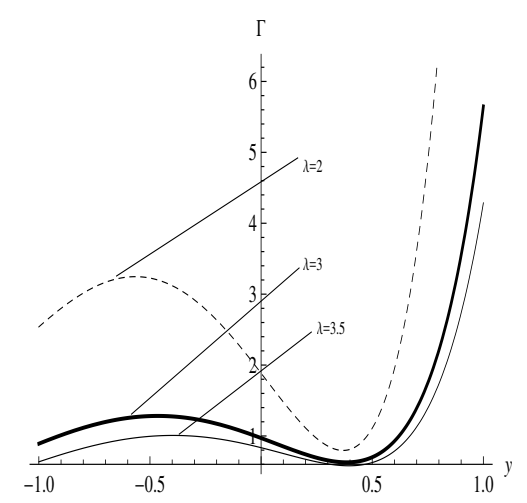

Figure 7: Effect of increasing couple stress inverse parameter on entropy generation rate 


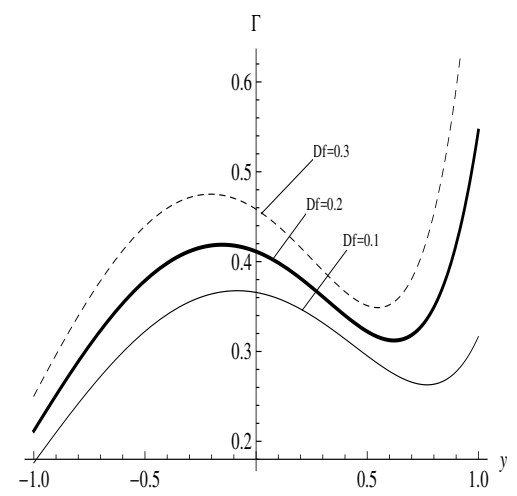

Figure 8: Effect of increasing Dufour number on entropy generation rate

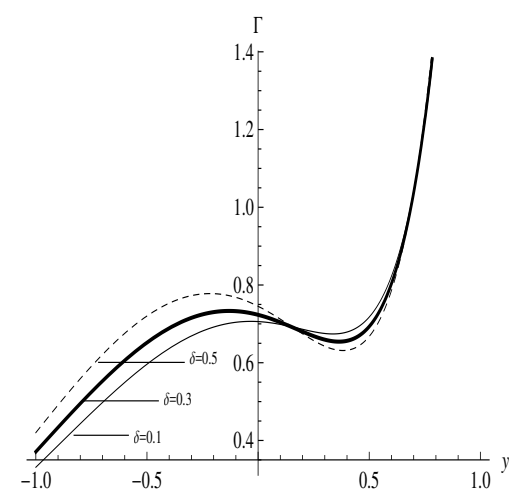

Figure 9: Effect of increasing internal heat generation parameter on entropy generation rate 


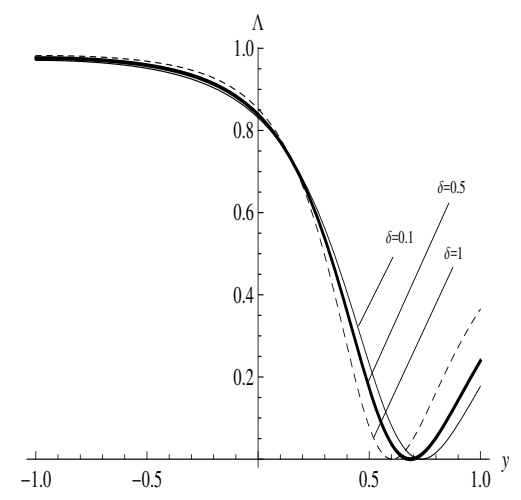

Figure 10: Effect of increasing internal heat generation parameter on Bejan number

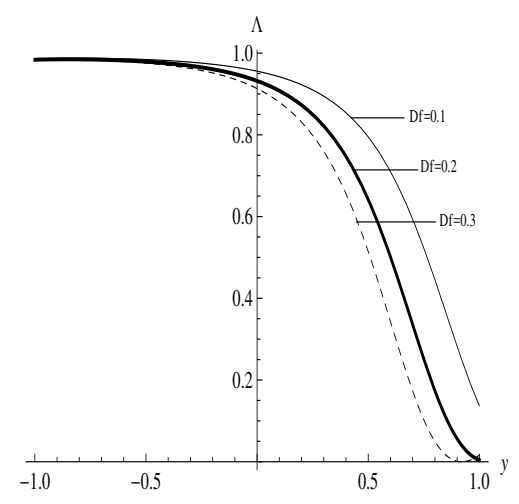

Figure 11: Effect of increasing Dufour number on Bejan number 


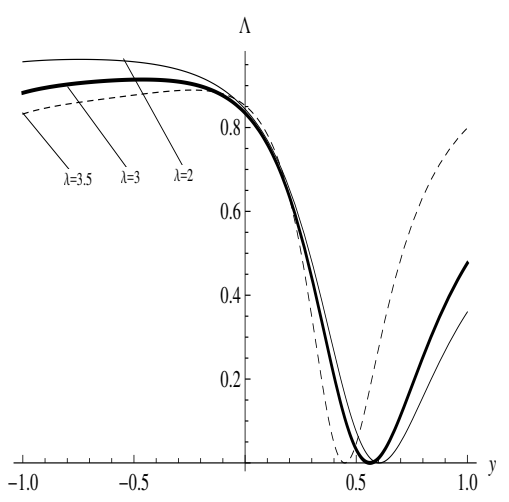

Figure 12: Effect of increasing couple stress inverse parameter on Bejan number

ilarly, in Figure 8, entropy generation increases with increase in Dufour number while entropy has minimal effect at the heated wall with increasing value of the internal heat generation parameter. However, entropy is observed to decrease in the region close to the heated wall. But at the cold wall, entropy generation increases significantly. As seen in figure 10, at the walls, heat transfer is observed to dominate the irreversibility of heat while fluid viscosity and concentration of the reacting fluid dominates the irreversibility within the channel. Figure 11 shows that as the Dufour number increases, over fluid friction and specie concentration dominates over the heat transfer. Finally, an increase in the couple stress inverse shows that specie concentration and fluid viscosity dominates the irreversibility while the reverse is the case at the heated wall.

\section{Conclusion}

In this work, a computational approach to study the entropy generation rate in the flow of a couple stress fluid with heat and mass transfer has been presented. Approximate solution of the couple boundary value problem is obtained using semi-analytical Adomian decomposition method. The main findings from the computation can be summarized as follows:

1. Increasing values of the fluid concentration and the internal heat generation has decreasing effect on the exergy of the thermal system. Therefore, a more efficient thermal system can be achieved if these parameter values are kept reasonably low. 
2. Increasing value of the couple stress parameter act as the entropy minimizer within the channel, this encourages the maximum performance of the system.

\section{Nomenclature}

$h \quad$ is the channel half width

$u^{\prime}, u \quad$ is the dimensionaland dimensionless axial velocity respectively,

$\mu \quad$ is the dynamic viscosity,

$P^{\prime} \quad$ is the fluid pressure,

$\rho \quad$ is the fluid density,

T, $\theta$ is the dimensional and dimensionless fluid temperature respectively,

$T_{0}, C_{0} \quad$ is the initial fluid temperature and concentration respectively,

$T_{1}, C_{1} \quad$ is the final fluid temperature and concentration respectively,

$k \quad$ is the thermal conductivity of the fluid,

$Q_{0}, \delta \quad$ heat source due to chemical reaction and internal heat generation parameter respectively

Sc Schmidt number

$Q_{1} \quad$ is the rate of chemical reaction

$\gamma \quad$ is the chemical reaction parameter

$C_{p} \quad$ is the specific heat at constant pressure,

$\nu_{0} \quad$ is the constant velocity of fluid suction/injection,

$\eta \quad$ is the fluid particle size effect due to couple stresses,

$E_{G} \quad$ is the local volumetric entropy generation rate

$R \quad$ is the suction/injection parameter,

$\lambda^{2} \quad$ is the couple stress parameter,

$\operatorname{Pr} \quad$ is the Prandtl number,

$\mathrm{Br} \quad$ is the Brinkman number,

$\Omega \quad$ is the parameter that measures the temperature difference between the two heat reservoirs

$\Gamma \quad$ is the dimensionless entropy generation rate.

$\phi \quad$ is the irreversibility distribution ratio.

$\Delta \quad$ Bejan number

$\beta_{T}, \beta_{C} \quad$ is the coefficient of thermal and solutal expansion

$g \quad$ is the acceleration due to gravity

$D_{M}, D_{f}$ is the solutal diffusivity of the medium and Dufour number respectively

$C, \varphi \quad$ is the dimensional and dimensionless concentration of the fluid respectively 


\section{References}

[1] A. Bejan, Second-law analysis in heat transfer and thermal design, Adv. Heat Trans., 15 (1982), 1-58.

[2] K. Hooman, F. Hooman and S.R. Mohebpour, Entropy generation for forced convection in a porous channel with isoflux or isothermal walls, International Journal of Exergy, $\mathbf{5 1}$ (2008), 78-96.

[3] S. Chen, Z. Liu, J. Liu, J. Li, L. Wang, C. Zheng, Analysis of entropy generation in hydrogen enriched ultra-lean counter-flow methane - air non-premixed combustion, Int. J. Hydrogen Energy, 35 (2010), 12491-12501.

[4] S. Chen, R. Du, Entropy generation of turbulent double-diffusive natural convection in a rectangle cavity, Energy, 36 (2011), 1721-1734.

[5] S. Chen, C. Zheng, Entropy generation in impinging flow confined by planar opposing jets, Int. J. Therm. Sci., 49 (2010), 2067-2075.

[6] A.O. Ajibade, B.K. Jha, A. Omame, Entropy generation under the effect of suction and injection, Applied Mathematical Modelling, 35 (2011), 4630-4046.

[7] S. Mahmud, R.A. Fraser, Mixed convection-radiation interaction in a vertical porous channel: Entropy generation, Energy, 28 (2003), 1557-1577.

[8] S.M. Tasnim, S. Mahmud, M.A.H. Mamum, Entropy generation in a porous channel with hydromagetic effect, Int. J. Exergy, 3 (2002), 300-308.

[9] O.D. Makinde, A.S. Eegunjobi, Entropy generation in a couple stress fluid flow through a vertical channel filled with saturated porous media, Entropy, 15 (2013), 4589-4606.

[10] S.O. Adesanya, O.D. Makinde, Entropy generation in couple stress fluid flow through porous channel with fluid slippage, International Journal of Exergy, Inderscience publishers (in press).

[11] S.O. Adesanya, O.D. Makinde, Effects of couple stresses on entropy generation rate in a porous channel with convective heating, Computational and Applied Mathematics, doi: 10.1007/s40314-014-0117-z.

[12] D. Srinivasacharya, K. Kaladhar, Soret and Dufour effects on free Convection flow of a couple stress fluid in a vertical channel with chemical reaction, Chemical Industry \& Chemical Engineering Quarterly, 19, No. 1 (2013), 45-55.

[13] R. Rajaram and M. Najafi, Analytical treatment and convergence of the Adomian decomposition method for a system of coupled damped wave equations, Applied Mathematics and Computation, 212 (2009), 72-81.

[14] M.M. Hosseini, H. Nasabzadeh, On the convergence of Adomian decomposition method, Applied Mathematics and Computation, 182 (2006), 536-543.

[15] I.L. El-Kalla, Convergence of the Adomian method applied to a class of nonlinear integral equations, Applied Mathematics Letters, 21 (2008), 372-376.

[16] E. Az-Zobi, K. Al-Khaled, A new convergence proof of the Adomian decomposition method for a mixed hyperbolic elliptic system of conservation laws, Applied Mathematics and Computation, 217 (2010), 4248-4256.

[17] S.O. Adesanya, Linear stability analysis of a plane-poiseuille hydromagnetic flow using Adomian decomposition method, U.P.B. Sci. Bull., Series A, 75 (2013), 99-106. 